\title{
Rossby Waves with Continuous Stratification and Bottom Friction
}

\author{
K. H. BRINK ${ }^{\mathrm{a}}$ AND J. PEDLOSKY \\ Woods Hole Oceanographic Institution, Woods Hole, Massachusetts
}

(Manuscript received 5 April 2018, in final form 26 July 2018)

\begin{abstract}
Published observations of subinertial ocean current variability show that the vertical structure is often well described by a vertical mode that has a node of horizontal velocity at the bottom rather than the traditional node of vertical velocity. The theory of forced and free linear Rossby waves in a continuously stratified ocean with a sloping bottom and bottom friction is treated here to see if frictional effects can plausibly contribute to this phenomenon. For parameter values representative of the mesoscale, bottom dissipation by itself appears to be too weak to be an explanation, although caution is required because the present approach uses a linear model to address a nonlinear phenomenon. One novel outcome is the emergence of a short-wave, bottomtrapped, strongly damped mode that is present even with a flat bottom.
\end{abstract}

\section{Introduction}

Study of observed subinertial ocean current variability shows a tendency for vertical structures not to fit a simple single baroclinic modal structure. For example, Wunsch (1997) showed that in many cases, both the barotropic and first baroclinic modes are present and that they are sometimes phase locked in the sense that enhances surface currents and thus weakens nearbottom currents (and sometimes in the opposite sense as well). Sanchez de la Lama et al. (2016, hereinafter SLF16) more recently summarized observed vertical structures using empirical orthogonal functions that very often have weak near-bottom velocities. Further, they showed that the variability is often wellrepresented by nontraditional ("rough bottom") baroclinic modes that have zero horizontal velocity at the bottom. Indeed, Samelson (1992) shows that a corrugated bottom can lead to weakened near-bottom currents, and SLF16 point out that a uniformly sloping bottom (e.g., Rhines 1970) can, depending on propagation direction and wave scale, also lead to vertical structures with weakened deep currents-hence the expression "rough-bottom modes." However, SLF16 show that there is not a clear statistical relationship between observed bottom roughness and modified

a ORCID: 0000-0001-9911-8807.

Corresponding author: K. H. Brink, kbrink@whoi.edu modal structure, a finding that LaCasce (2017) rationalizes in terms of the ubiquity of bottom slopes.

It seems timely to ask whether bottom friction might play a role in weakening deep ocean currents. For example, simple two-layer linear wave problems show that friction decreases deep currents at low frequency (e.g., Allen 1984), and various numerical calculations of nonlinear flows demonstrate that bottom friction also affects the vertical structure of eddy processes (e.g., LaCasce and Brink 2000; Arbic and Flierl 2004; Trossman et al. 2017; Brink 2017). However, it is not completely clear how bottom friction will affect traditional baroclinic modes per se. The present study thus addresses the topic of how strongly bottom friction affects baroclinic modal structures. The question is approached in the context of linear Rossby waves in a continuously stratified ocean.

\section{Methodology and a calculation}

\section{a. Formulation}

The linear quasigeostrophic vorticity equation is

$$
Q=p_{x x t}+p_{y y t}+\beta p_{x}+f^{2}\left(\frac{p_{z t}}{N^{2}}\right)_{z}
$$

where $p$ is pressure, $f$ is the Coriolis parameter, $\beta$ is the northward gradient of $f, N$ is the buoyancy frequency, and $Q$ represents an unspecified potential vorticity source. The $(x, y, z)$ coordinates are eastward, 
northward, and vertical, while $t$ is time. Subscripted independent variables represent partial differentiation. The equation is to be solved subject to a rigid lid and (assuming infinitesimally thin turbulent boundary layers) an Ekman compatibility surface condition:

$$
\frac{p_{z t}}{N^{2}}=-\left(\tau_{0 x}^{y}-\tau_{0 y}^{x}\right) f^{-1} \quad \text { at } \quad z=0
$$

where $\left(\tau_{0}^{x}, \tau_{0}^{y}\right)$ is the wind stress vector. At the sloping bottom, there is also an Ekman compatibility condition:

$$
\begin{aligned}
\frac{p_{z t}}{N^{2}} & =-\alpha^{x} f^{-1} p_{y}+\alpha^{y} f^{-1} p_{x}-r\left(p_{x x}+p_{y y}\right) f^{-2} \text { at } \\
z & =-h_{0},
\end{aligned}
$$

where $\alpha^{x}$ and $\alpha^{y}$ are the bottom slopes in the $x$ and $y$ directions, respectively (i.e., $h=h_{0}+\alpha^{x} x+\alpha^{y} y$, with $\left.\left|\alpha^{x} x\right|,\left|\alpha^{y} y\right| \ll h_{0}\right)$, and $r$ is the effective bottom resistance coefficient. In the following, the bottom will often be taken to be flat so that $\alpha^{x}=\alpha^{y}=0$.

\section{b. Vertical modal solutions}

The solution to (1) for free mode $n$ is taken to have the form

$$
p=F_{n}(z) \exp \left[i\left(k x+l y+\omega_{n} t\right)\right]
$$

so that [from (1)]

$$
0=\left(\frac{F_{n z}}{N^{2}}\right)_{z}+\mu_{n}^{2} F_{n},
$$

where

$$
-\mu_{n}^{2} f^{2}=k^{2}+l^{2}-\frac{\beta k}{\omega_{n}} .
$$

The unforced boundary conditions are

$$
\begin{aligned}
& F_{n z}=0 \quad \text { at } \quad z=0 \text { and } \\
& \frac{F_{n z}}{N^{2}}=\frac{1}{\omega_{n} f} \Gamma F_{n} \quad \text { at } \quad z=-h_{0},
\end{aligned}
$$

where

$$
\Gamma=-\alpha^{x} l+\alpha^{y} k-\frac{i r}{f}\left(k^{2}+l^{2}\right) .
$$

When the stratification is constant, $N^{2}=N_{0}^{2}$, the solution takes the simple form of

$$
F_{n}=b \cos \left(\zeta_{n} z\right)
$$

$$
\zeta_{n}=\mu_{n} N
$$

where application of (5) leads to

$$
\zeta_{n} \tan \left(\zeta_{n} h_{0}\right)=A+B \zeta_{n}^{2}
$$

with

$$
\begin{aligned}
A & =\Gamma \frac{\left(k^{2}+l^{2}\right) N_{0}^{2}}{\beta k f}, \\
B & =\frac{\Gamma f}{\beta k} .
\end{aligned}
$$

Analytical approximate solutions to this problem are detailed in sections $2 \mathrm{~d}$ and $2 \mathrm{e}$ below. The modal functions have the familiar property of no vertical gradient (i.e., no vertical velocity) at the surface, while at depth, higher modes have increasingly sinuous structure, but always with the vertical velocity (hence $F_{n z}$ ) nonzero at the top of the infinitesimal bottom boundary layer $\left(z=-h_{0}\right)$ as long as $\Gamma \neq 0$, for example, in order to accommodate Ekman pumping when $r \neq 0$. Thus, the symmetry of the upper and bottom boundary conditions is broken. Further, when bottom friction is nonzero, $\Gamma$, $\zeta_{n}, \omega_{n}$, and $F_{n}$ are complex and wave phase is no longer uniform in the vertical.

\section{c. A nondimensional parameter}

A scaling of the bottom boundary condition [(5b)] helps reveal when frictional effects cease to be perturbations and begin to revamp the wave modal structures. Specifically, the time derivative in (2b) is estimated with the inviscid barotropic Rossby wave frequency $\omega_{0 R}$ (the largest frequency, or shortest time scale, available for any wavenumber pairing). Thus, a nondimensional parameter quantifying the frictional effect is

$$
R=\frac{r}{\left(\omega_{0 R} h_{0}\right)} \frac{N_{B}^{2} h_{0}^{2}}{f^{2}}\left(k^{2}+l^{2}\right)
$$

where $N_{B}$ is the buoyancy frequency at the bottom $\left(z=-h_{0}\right)$. This expression can be thought of as consisting of two ratios. The first, $r\left(h_{0} \omega_{0 R}\right)^{-1}$, is the ratio of the wave time scale to the barotropic spindown time, a measure of the overall importance of bottom friction. The remaining factors in (7) constitute a Burger number for the wave, that is, the ratio of the natural vertical scale in a stratified system (the deformation scale) to the water depth. If the deformation scale is large relative to the water depth, frictional effects are distributed throughout the water column. If the deformation scale is less than the water depth, frictional effects will be trapped near the bottom. This interpretation 
emphasizes that frictionally adjusted modal structures depend on both the relative strength of damping and the relative importance of the stratification at a given length scale. Thus, for $R \ll 1$, modal structures are unperturbed by bottom friction, but for $R \geq O(1)$, frictional effects lead to substantial modal adjustments.

An expression similar to (7) can be derived by multiplying (4a) by $F_{n}$ and integrating over depth. The result is

$$
\mu_{n}^{2} \int_{-h}^{0} F_{n}^{2} d z=\int_{-h}^{0} \frac{F_{n z}^{2}}{N^{2}} d z+\frac{\Gamma}{f \omega_{n}} F_{n}(-h)^{2} .
$$

Comparing the two terms on the right-hand side, it is found that frictional modification of modal structure over a flat bottom becomes important when

$$
R_{I}=\frac{r}{\left(\omega_{0 R} h_{0}\right)} \frac{\overline{N^{2}} h_{0}^{2}}{f^{2}}\left(k^{2}+l^{2}\right)
$$

becomes $O(1)$, where $\overline{N^{2}}$ is a depth average. While this expression may not be as intuitive as that leading to (7), it has the advantage of applying to arbitrary distributions of the stratification. This is a concern because the usefulness of (7) is questionable when $N_{B}$ is extremely small, as is the case with an exponential stratification.

At first, it may be unintuitive that the frictional effect increases with stratification. After all, stratification inhibits the vertical velocity that is the messenger of spindown in the water column. This is indeed the case, but stronger stratification also leads to an increasing tendency to trap any spindown processes closer to the bottom. Further, stronger stratification, by trapping spindown closer to the bottom, can inhibit near-bottom horizontal flow and hence decrease bottom stresses. Thus, since the bottom boundary condition $[(5 b)]$ deals only with conditions very near the bottom, there is no contradiction. Moreover, the frictional modifications to the vertical modal structure often serve to decrease wave damping, as will be seen below.

\section{d. Asymptotics: Barotropic and bottom-trapped modes}

With constant $N^{2}=N_{0}^{2}$, a solution to (4a) subject to (5a) can take the form

$$
F=a \cosh (\gamma z),
$$

where

$$
\gamma=\frac{N_{0}}{f}\left(k^{2}+l^{2}-\frac{\beta k}{\omega}\right)^{1 / 2}
$$

allows solutions for $F$ that are either barotropic or bottom intensified. [Choosing this hyperbolic cosine form in (9a) - as opposed to a simple cosine form-is somewhat arbitrary, since $\gamma$ is complex, but treating the forms separately is thought to improve clarity. This is equivalent to choosing $\operatorname{Real}\left(\mu_{n}^{2}\right)<0$ in (4b).] Substitution of (9a) into the bottom boundary condition in $(5 b)$ yields

$$
\omega=\frac{i r\left(k^{2}+l^{2}\right)}{\gamma \tanh \left(\gamma h_{0}\right)} \frac{N_{0}^{2}}{f^{2}} .
$$

If stratification is weak, or if $\omega$ falls near the inviscid barotropic Rossby wave resonance, $\left|\gamma h_{0}\right| \ll 1, \tanh \left(\gamma h_{0}\right) \approx$ $\gamma h_{0}$, and (10) simply reduces to

$$
\omega \approx \frac{\beta k}{k^{2}+l^{2}}+\frac{i r}{h_{0}}
$$

with no further approximations. This same form would result from assuming at the outset that there is no stratification.

On the other hand, for stronger stratification, $\operatorname{Re}(\gamma) h_{0} \gg$ 1 , bottom trapping occurs and $\tanh \left(\gamma h_{0}\right) \rightarrow 1$. Thus, (10) becomes

$$
\omega \gamma=i r\left(k^{2}+l^{2}\right) \frac{N_{0}^{2}}{f^{2}}
$$

so that

$$
0=\omega^{2}-\left(\frac{\beta k}{k^{2}+l^{2}}\right) \omega+r^{2}\left(k^{2}+l^{2}\right) \frac{N_{0}^{2}}{f^{2}} .
$$

When $R$ becomes large, the physically realizable (decaying) solution to (13) is then

$$
\omega=\operatorname{ir}\left(k^{2}+l^{2}\right)^{1 / 2} \frac{N_{0}}{f} .
$$

A physical interpretation of this scale is that it represents the inverse spindown time of a region having vertical extent $f\left[N_{0}\left(k^{2}+l^{2}\right)^{1 / 2}\right]^{-1}$.

\section{e. Asymptotics: Baroclinic modes}

Solutions to (4a) with constant stratification subject to (5a) can be written in the form

$$
F(z)=b \cos (\eta z)
$$

where

$$
\eta^{2}=\frac{N_{0}^{2}}{f^{2}}\left(\frac{\beta k}{\omega}-k^{2}-l^{2}\right)
$$


is a complex quantity. The form (15a) is equivalent to choosing $\operatorname{Real}\left(\mu_{n}^{2}\right)>0$ in (4b). The parameter $\eta$ takes on discrete values in accord with the bottom boundary condition, and so

$$
\omega=\frac{\beta k}{k^{2}+l^{2}+f^{2} N_{0}^{-2} \eta^{2}} .
$$

For small $R$, an approximate solution is found by seeking a perturbation around the $r=0$ values:

$$
\eta^{(0)}=n \pi h_{0}^{-1}, \quad n=1,2,3, \ldots
$$

Assuming small, $O(R)$ corrections, the expansions

$$
\eta \approx \eta^{(0)}+\eta^{(1)}
$$

and

$$
\omega \approx \omega^{(0)}+\omega^{(1)}
$$

(where $\omega^{(0)}$ is the frictionless frequency) are used. Thus, (5b) yields

$i\left(\omega^{(0)}+\omega^{(1)}\right) \tan \left[\left(\eta^{(0)}+\eta^{(1)}\right) h_{0}\right]=\frac{r N_{0}^{2}}{\left(\eta^{(0)}+\eta^{(1)}\right) f^{2}}\left(k^{2}+l^{2}\right)$

and, after Taylor expansion and using $\tan \left(\eta^{(0)} h_{0}\right)=0$,

$$
\eta^{(1)} \cong-i r N_{0}^{2} f^{-2} h_{0}^{-1}\left(k^{2}+l^{2}\right)\left(\omega^{(0)} \eta^{(0)}\right)^{-1} ;
$$

that is, the correction to the vertical trapping scale increases with the square of scalar wavenumber, as might be expected from (7). Using this form in (16) and expanding $\eta^{2} \approx \eta^{(0) 2}+2 \eta^{(0)} \eta^{(1)}$ leads to the solution

$$
\omega^{(1)}=\frac{i 2 r\left(k^{2}+l^{2}\right) h_{0}^{-1}}{k^{2}+l^{2}+\eta^{(0) 2} \frac{f^{2}}{N_{0}^{2}}},
$$

for small $R$ (i.e., long waves or weak friction).

In the limit of large $R$, a similar expansion is carried out for small $R^{-1}$. In this case, we anticipate that interior velocity near the bottom becomes weak (see section $2 \mathrm{f}$ below) so that $v \rightarrow 0$ at $z=-h_{0}$. Thus,

$$
\eta^{(0)}=(2 n-1) \pi\left(2 h_{0}\right)^{-1} \text { for } n=1,2,3, \ldots
$$

Then, the correction (imaginary) frequency is

$$
\omega^{(1)}=i 2 \frac{\omega^{(0) 2} \eta^{(0) 2}}{r h_{0}} \frac{f^{4}}{N_{0}^{4}}\left[\left(k^{2}+l^{2}\right)\left(k^{2}+l^{2}+\frac{f^{2} \eta_{0}^{2}}{N_{0}^{2}}\right)\right]^{-1}
$$

and

$$
\eta^{(1)}=-i \omega^{(0)} \eta^{(0)} f^{2}\left[r N_{0}^{2} h_{0}\left(k^{2}+l^{2}\right)\right]^{-1}
$$

for large $R$.

\section{f. Computed results}

When there is no bottom friction, $\Gamma$ is real and it is straightforward to obtain the infinity of solutions for $F_{n}$ by solving (6c) for constant stratification. From a practical standpoint, however, solutions of the form (6a) with $r \neq 0$ raise computational difficulties because solving (6c) involves searches in the complex plane, such that there is sometimes a question as to whether all of the desired roots have been found. For example, it is usually easy to find the solution having the simplest vertical structure, but then some higher-mode solutions can be harder to isolate. A messier, but more algorithmically certain, approach is to expand $F_{n}$ in terms of the complete set of inviscid, flat-bottom baroclinic modes and thus replace (6) with an algebraic eigenvalue problem that is straightforward to solve with readily available software.

For the case of constant $N^{2}=N_{0}^{2}$, the inviscid, flatbottom (where $\Gamma=0$ ) modes are

$G_{m}=a_{m} \cos \left(m \pi z h_{0}^{-1}\right)$, where $m=0,1,2, \ldots$.

It is also well known that analytical expressions for $\Gamma=0$ modal structures can also be found for exponential stratification:

$$
\begin{aligned}
& N^{2}=N_{S}^{2} \exp \left(z z_{S}^{-1}\right), \text { and } \\
& G_{m}=a_{m}\left[-\frac{Y_{0}\left(q_{m B}\right)}{J_{0}\left(q_{m B}\right)} J_{1}\left(q_{m}\right)+Y_{1}\left(q_{m}\right)\right] \exp \left(0.5 z z_{S}^{-1}\right)
\end{aligned}
$$

where $J_{0}, J_{1}, Y_{0}$, and $Y_{1}$ are Bessel functions, $a_{m}$ is a constant,

$$
q_{m}=\lambda_{m} \exp \left(0.5 z z_{S}^{-1}\right),
$$

$q_{m B}$ is $q_{m}$ evaluated at $z=-h_{0}$, and the multiple values of $\lambda_{m}$ are found by solving

$$
\begin{aligned}
0= & J_{0}\left(\lambda_{m}\right) Y_{0}\left[\lambda_{m} \exp \left(-0.5 h_{0} z_{S}^{-1}\right)\right] \\
& -Y_{0}\left(\lambda_{m}\right) J_{0}\left[\lambda_{m} \exp \left(-0.5 h_{0} z_{S}^{-1}\right)\right] .
\end{aligned}
$$

In either case, the modes are normalized so that

$$
1=\int_{-h_{0}}^{0} G_{m}^{2} d z
$$


The unforced problem for $\Gamma \neq 0$ is then solved by expressing the solution in terms of the inviscid modes; namely,

$$
F_{n}(z)=\sum_{m=0}^{\Lambda} b_{n m} G_{m}(z)
$$

where $\Lambda$ is a suitably large integer $(\Lambda=50$ or more in the following calculations), and the notation $F_{n}$ anticipates the multiple solutions to the problem. Multiplication of (4a) by $G_{m}$, followed by vertical integration and application of (5a) and (5b) then yields an algebraic eigenvalue problem for $\omega_{n}$ :

$$
0=\left(\omega_{n}-D_{m}\right) b_{n m}+f \Gamma \sum_{q} b_{n q} E_{m}^{-1} G_{m}\left(-h_{0}\right) G_{q}\left(-h_{0}\right)
$$

(for $n=1,2,3, \ldots, \Lambda)$, where

$$
\begin{aligned}
& D_{m}=\beta k E_{m}^{-1}, \\
& E_{m}=k^{2}+l^{2}+f^{2} c_{m}^{-2},
\end{aligned}
$$

and $c_{m}$ is the long gravity wave speed associated with inviscid mode $m$. For example, for constant stratification,

$$
c_{m}=N_{0} h_{0}(m \pi)^{-1}
$$

and for exponential stratification [(25a)],

$$
c_{m}=2 z_{S} N_{S} \lambda_{m}^{-1} .
$$

The problem in (28a) is solved using a MATLAB function, and the eigenvalues and eigenvectors having the largest absolute values of $\omega_{n}$ are saved. The first three solutions are identified as $\omega_{0}, \omega_{1}$, and $\omega_{2}$, and each is associated with a set $b_{0 m}, b_{1 m}$, and $b_{2 m}$. Using these, the frictional modal structures $F_{0}, F_{1}$, and $F_{2}$ are readily constructed.

A sample calculation with constant $N^{2}$, a flat bottom, and representative parameters illustrates the frictional effects. Specifically, $N_{0}^{2}=2.58 \times 10^{-6} \mathrm{~s}^{-2}, h_{0}=4500 \mathrm{~m}$, $l=2 \times 10^{-6} \mathrm{~m}^{-1}, r=1 \times 10^{-4} \mathrm{~m} \mathrm{~s}^{-1}, f=0.73 \times$ $10^{-4} \mathrm{~s}^{-1}, \beta=2 \times 10^{-11}(\mathrm{~s} \mathrm{~m})^{-1}$, and $k$ is varied over a wide range. The $N_{0}^{2}$ is chosen so that $c_{1}=2.3 \mathrm{~m} \mathrm{~s}^{-1}$ in (29a), while $f$ and $\beta$ are representative of $30^{\circ}$ latitude. Because $r$ depends on the strength of total bottom currents (including tides; e.g., Wright and Thompson 1983), it is not obvious what an appropriate deep-sea value might be, but $r=5 \times 10^{-4} \mathrm{~m} \mathrm{~s}^{-1}$ is commonly used over the continental shelf (e.g., Chapman 1987), and one might expect abyssal currents in most of the
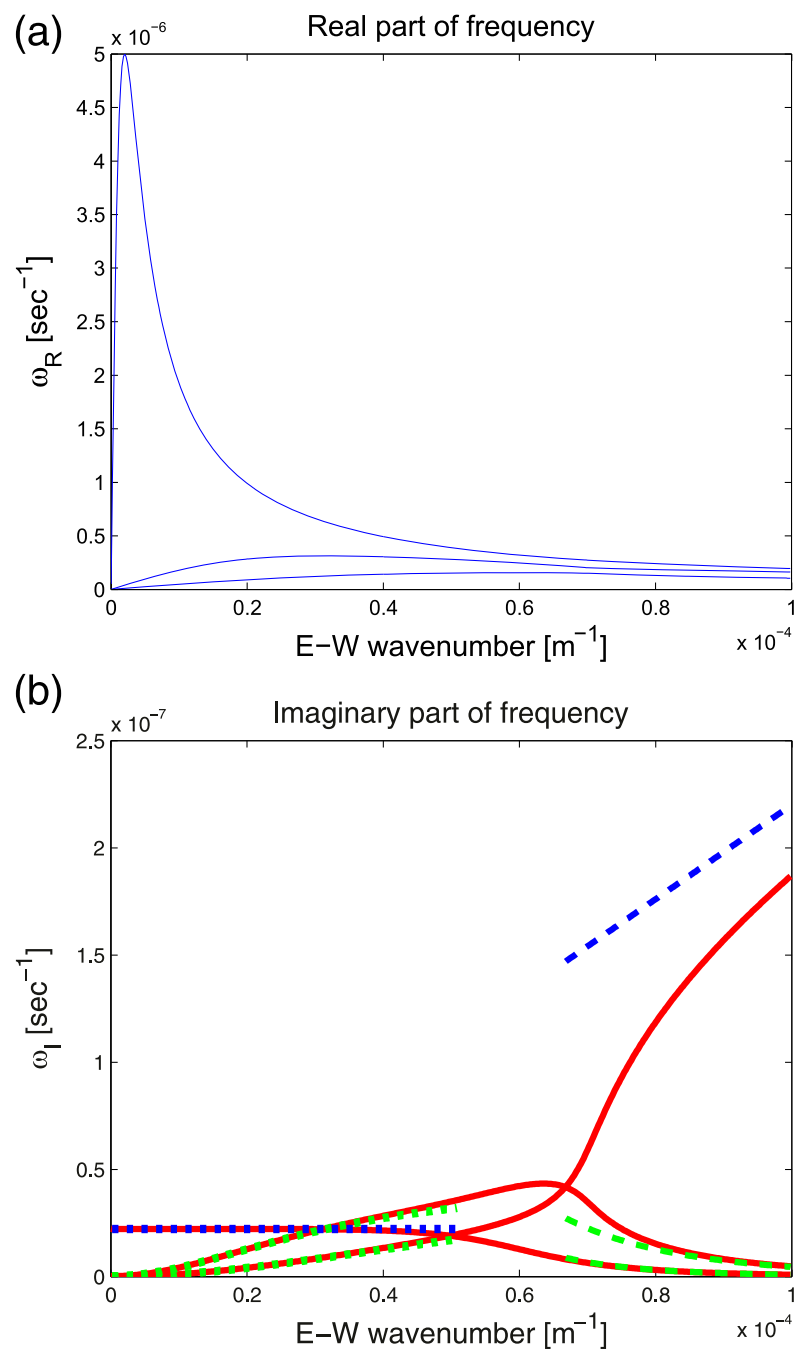

FIG. 1. Frequency vs east-west wavenumber $k$ for Rossby waves with constant $N^{2}$. (a) Real part of frequency $\omega_{R}$ and (b) imaginary part of frequency $\omega_{I}$ (in red, with asymptotic expressions as blue or green broken lines). The blue curves are for modes that are barotropic or bottom intensified (section $2 \mathrm{~d}$ ), and the green curves are for baroclinic modes (section 2e). Expressions for small $k$ are dotted lines and for large $k$ are dashed lines. Computed with $N_{0}^{2}=2.58 \times 10^{-6} \mathrm{~s}^{-2}, h=4500 \mathrm{~m}, l=2 \times 10^{-6} \mathrm{~m}^{-1}, r=1 \times$ $10^{-4} \mathrm{~m} \mathrm{~s}^{-1}, f=0.73 \times 10^{-4} \mathrm{~s}^{-1}$, and $\beta=2 \times 10^{-11}(\mathrm{~s} \mathrm{~m})^{-1}$. Note that the vertical axes have different scales in the two panels.

ocean to be weaker than representative shelf conditions. Hence the choice of $r=1 \times 10^{-4} \mathrm{~m} \mathrm{~s}^{-1}$ for these calculations.

Results of the calculation are summarized in the dispersion curves of Fig. 1. For small $k$, the right-hand side of $(2 b)$ is small (i.e., the curl of the bottom stress is small), so that frictional effects are weak, and the modal structures (Fig. 2) are essentially $G_{n}$, that is, the forms found in the complete absence of bottom friction. The smaller imaginary part of the modal structure 


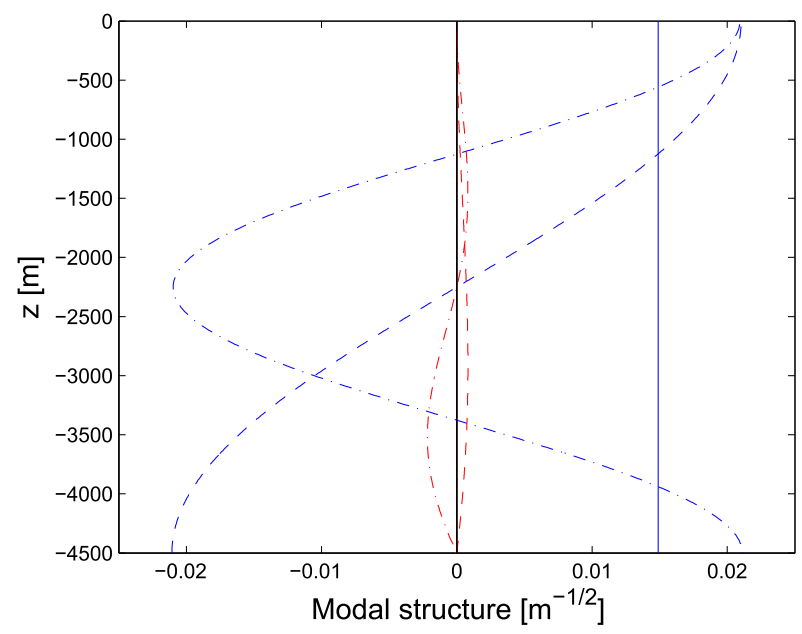

FIG. 2. Long-wave vertical modal structures for conditions as in Fig. 1 and $k=2.1 \times 10^{-7} \mathrm{~m}^{-1}$. The wave frequencies are as follows: $\omega_{0}($ solid curves $)=1.04 \times 10^{-6}+i 2.22 \times 10^{-8} \mathrm{~s}^{-1}, \omega_{1}($ dashed $)=$ $4.15 \times 10^{-9}+i 1.78 \times 10^{-10} \mathrm{~s}^{-1}$, and $\omega_{2}($ dash-dotted $)=1.04 \times$ $10^{-9}+i 4.49 \times 10^{-11} \mathrm{~s}^{-1}$. Blue lines indicate the real part of the modal structure, and red lines indicate the accompanying imaginary parts.

gives rise to phase shifts as a function of the vertical. The real part of frequency $\omega_{R}$ is essentially the inviscid result, while the imaginary part of frequency $\omega_{I}$ (Fig. 1b) behaves as one would expect from a perturbation expansion for small $r$ (see sections $2 \mathrm{~d}$ and $2 \mathrm{e}$ ). However, as $k$ increases, $\omega_{I}$ increases for two of the modes, and then $\omega_{I}$ for two modes decreases for larger $k$. At the same time, $\omega_{I}$ for the other mode abruptly begins to increase dramatically for $k>0.7 \times 10^{-4} \mathrm{~m}^{-1}$. What is happening? Examination of the modal structures for large $k$ (Fig. 3) clarifies the situation. Two of the modes adjust so that there is a node in horizontal velocity near the bottom; that is, they can be thought of as becoming modes $n=1 / 2$ and $n=3 / 2$. The boundary condition $[(2 b)$ or $(5 b)]$ is being met by having the bottom stress become small even though $r\left(k^{2}+l^{2}\right)$ is growing. This sort of behavior, where a linear wave mode structure adjusts so that the effect of bottom friction is minimized, is not unusual in oceanographic problems (e.g., Allen 1984; Power et al. 1989; Brink 2006). On the other hand, the wave mode that has damping increasing with $k$ has reached a state, for large $k$, that is strongly bottom trapped (Fig. 3). With a large bottom velocity, there is nothing to mitigate the growing $r\left(k^{2}+l^{2}\right)$, and so the wave damping grows strongly as $k$ increases and bottom trapping becomes more pronounced. In this case, stratification increases the wave damping because it leads to intensified (rather than weakened) nearbottom currents. It is worth pointing out that this bottom-trapping happens with a flat bottom.

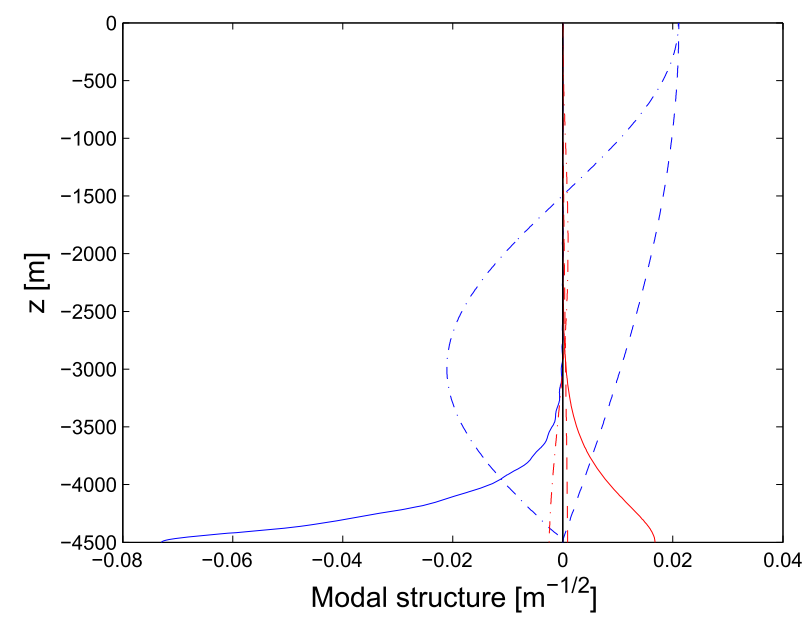

FIG. 3. Short-wave vertical modal structures for conditions as in Fig. 1 and $k=1.5 \times 10^{-4} \mathrm{~m}^{-1}$. The wave frequencies are: $\omega_{0}$ (solid curves $)=7.01 \times 10^{-8}+i 3.033 \times 10^{-7} \mathrm{~s}^{-1}, \omega_{1}$ (dashed) $=1.32 \times$ $10^{-7}+i 7.93 \times 10^{-11} \mathrm{~s}^{-1}$, and $\omega_{2}($ dash-dot $)=1.21 \times 10^{-7}+i 5.54 \times$ $10^{-10} \mathrm{~s}^{-1}$. Blue lines indicate the real part of the modal structure, and red lines indicate the accompanying imaginary parts.

The asymptotic expressions for the imaginary part of frequency for barotropic or bottom-trapped modes [(11) and (14)] are overplotted as blue broken lines in Fig. 1b. The weak-friction limit in (11) is seen to replicate the calculations quite well for $k<0.4 \times 10^{-4} \mathrm{~m}^{-1}$, while the strong-friction limit (the blue dashed line for $\omega_{I}>1.5 \times 10^{-7} \mathrm{~s}^{-1}$ ) captures only the right magnitude and trend in this example.

The asymptotic results for baroclinic modes [(21) and (23)] are overplotted as green broken lines in Fig. 1 b. They both provide excellent agreement with the direct calculations in the appropriate $R$ range. Note that three of the expressions for wave damping rates-(11), (14), and (21) — do not depend on wave orientation, although the large $R$, higher-mode expression in (23) does depend on orientation through $\omega^{(0)}$.

Up to this point, all results have been for the case of constant $N^{2}$. One might ask whether the results change substantially when one uses a more realistic, surfaceintensified exponential stratification. The inviscid baroclinic modes in this case obey (25), and we choose $z_{S}=$ $350 \mathrm{~m}$ (following SLF16) and $N_{S}^{2}=7.75 \times 10^{-5} \mathrm{~s}^{-2}$ in order to obtain a first internal mode gravity wave speed of $2.3 \mathrm{~m} \mathrm{~s}^{-1}$ as in the case with constant $N^{2}$. The resulting modal structures (such as the dashed line in the left panel of Fig. 5) have larger amplitudes near the surface and relatively constant values in the lower portions of the water column where $N^{2}$ is small. After solving (28), Fig. 4 is obtained for the imaginary part of the frequency. We emphasize that the only difference between this and the calculations leading to the lower panel of 


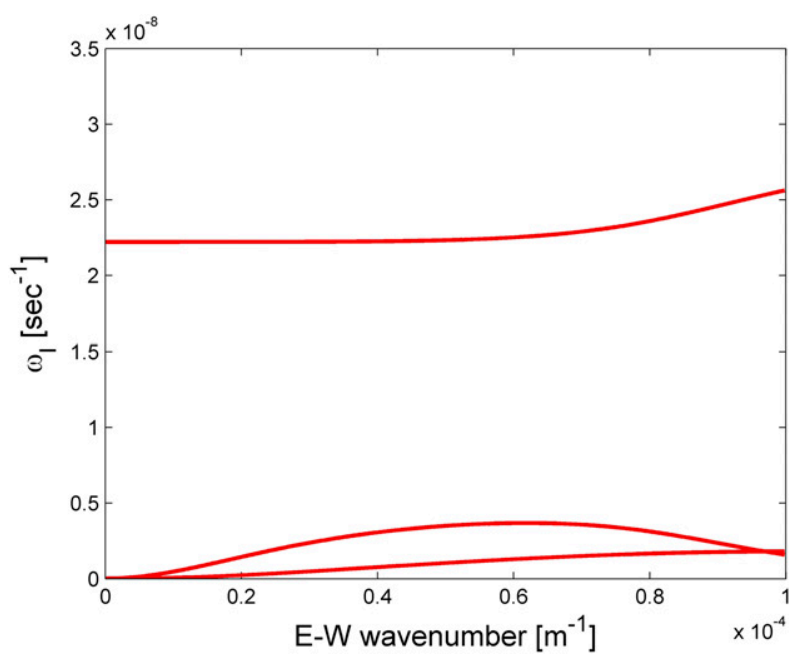

FIG. 4. Imaginary part of frequency for conditions as in Fig. 1, but the stratification is exponential $\left(N_{S}^{2}=7.75 \times 10^{-5} \mathrm{~s}^{-2} ; z_{S}=350 \mathrm{~m}\right)$. Note the change in vertical scale relative to Fig. $1 \mathrm{~b}$.

Fig. 1 is the use of exponential stratification. Two points stand out. First, the long-wave damping is much weaker (note the changed vertical scale) with exponential stratification than in the case for constant stratification. This is not surprising in light of the relatively weak (compared to the upper water column) bottom expression of the baroclinic modes with weak deep stratification. Second, the transition between inviscid modal structures (for longer waves) and the bottom- or surface-intensified large-friction (short wave) modes occurs at roughly the same wavelengths. For example, the long-wave barotropic wave's flat $\omega_{I}$ curve begins to bend at about $k=3 \times 10^{-5} \mathrm{~m}^{-1}$ with constant $N^{2}$ (Fig. 1) and around $k=4 \times 10^{-5} \mathrm{~m}^{-1}$ with the exponential stratification (Fig. 4). Similarly, the first baroclinic mode reaches its maximum $\omega_{I}$ at $k=6.4 \times 10^{-5} \mathrm{~m}^{-1}$ for constant $N^{2}$ (Fig. 1) and at $6.2 \times 10^{-5} \mathrm{~m}^{-1}$ for exponential stratification (Fig. 4). We conclude from this insensitivity that the vertical structure of the density stratification does not substantially affect either 1) our conclusions about the importance of bottom friction for changing modal structures or 2) the utility of the $R_{I}$ parameter [(8)]. [It is evidently more appropriate to use a depth-averaged $N^{2}$ than the actual near-bottom value (7).]

The results presented here are fairly representative of many calculations (not detailed here), executed with both constant and exponential $N^{2}$, in that, in all cases, most wave modes evolve toward a state with weak near-bottom velocities and with decreasing wave damping as $r\left(k^{2}+l^{2}\right)$ increases. However, in every calculation, there is always one wave mode that is increasingly bottom intensified and where the damping becomes large. In some calculations, the bottomtrapped wave mode is continuous with the inviscid barotropic mode as $k$ varies (e.g., Fig. 4), but in some cases (such as Fig. 1), the bottom-trapped mode is continuous with one of the small- $r$ baroclinic modes. Also, for a given set of parameters, the transition from the nearly inviscid modal structure toward the strongly frictional structures tends to occur at roughly the same wavenumber range (e.g., where $k \approx 0.7 \times 10^{-4} \mathrm{~m}^{-1}$ and $R_{I}=3.7$ for Fig. 1b) for each of the three gravest modes. This transition occurs where the deformation scale $\left[\approx 2 \pi f /\left(N_{0} k\right)=4100 \mathrm{~m}\right.$ here $]$ is comparable to the ocean's $4500-\mathrm{m}$ depth. Finally, even with a sloping bottom $\left(\alpha^{x}\right.$ and/or $\alpha^{y} \neq 0$ ), the results do not change qualitatively: for large $r\left(k^{2}+l^{2}\right)$, there is a single, strongly damped, bottom-trapped wave mode while all other modes adjust so that near-bottom velocity is small.

\section{Forced solutions}

Assuming that variables are all horizontally harmonic in space,

$$
\begin{aligned}
p & =P(z, t) \exp [i(k x+l y)], \\
Q & =\hat{Q}(z, t) \exp [i(k x+l y)], \quad \text { and } \\
\left(\tau_{0}^{x}, \tau_{0}^{y}\right) & =\left(\hat{\tau}_{0}^{x}, \hat{\tau}_{0}^{y}\right) \exp [i(k x+l y)],
\end{aligned}
$$

the forced problem in (1) and (2) can be stated as

$$
\begin{aligned}
\hat{Q} & =-\left(k^{2}+l^{2}\right) P_{t}+i \beta k P+f^{2}\left(\frac{P_{z t}}{N^{2}}\right)_{z} \\
\left(\frac{P_{z t}}{N^{2}}\right) & =-i f^{-1}\left(\hat{\tau}_{0}^{y} k-\hat{\tau}_{0}^{x} l\right) \quad \text { at } \quad z=0, \quad \text { and } \\
\left(\frac{P_{z t}}{N^{2}}\right) & =\left[r f^{-2}\left(k^{2}+l^{2}\right)+i f^{-1}\left(\alpha^{y} k-\alpha^{x} l\right)\right] P \quad \text { at } \\
z & =-h_{0}
\end{aligned}
$$

Similarly, the problem for the vertical modes can be expressed for general $N^{2}$ as (4a) and (5) so that the (generally complex) free mode frequency for mode $n$ is given by

$$
\omega_{n}=\frac{\beta k}{k^{2}+l^{2}+f^{2} \mu_{n}^{2}} .
$$

It is straightforward to show that the vertical modes are orthogonal according to

$$
\delta_{n m}=\int_{-h_{0}}^{0} F_{n} F_{m} d z-\frac{f \Gamma}{\beta k} F_{n}\left(-h_{0}\right) F_{m}\left(-h_{0}\right),
$$


where $\delta_{n m}$ is the Kronecker delta ( $=1$ for $n=m$ and $=0$ for $n \neq m)$. Bear in mind that the function $\Gamma$ is complex when $r \neq 0$, and even when $r=0$, it can be either positive or negative. Note that the normalization implied by this condition reduces to a more traditional form [(26)] when $\Gamma=0$, that is, when the bottom is flat and there is no dissipation.

To proceed, multiply (31a) by $F_{n}$ and (4a) by $f^{2} P_{t}$ and subtract and integrate over depth. After applying boundary conditions [(5a) and (5b) and (31b) and (31c)], the following expression is obtained:

$$
\begin{aligned}
\text { if }(\beta k)^{-1} F_{n}(0)\left(k \hat{\tau}_{0}^{y}-l \hat{\tau}_{0}^{x}\right)+(\beta k)^{-1} \int_{-h_{0}}^{0} F_{n} \hat{Q} d z= & \int_{-h_{0}}^{0}\left(i P-\omega_{n}^{-1} P_{t}\right) F_{n} d z-f(\beta k)^{-1} \Gamma F_{n}\left(-h_{0}\right) \\
& \times\left[i P\left(-h_{0}, t\right)-\omega_{n}^{-1} P_{t}\left(-h_{0}, t\right)\right] .
\end{aligned}
$$

Now, expand the solution for $P$ in terms of the modes $F_{m}$ :

$$
P(z, t)=\sum_{m} \varphi_{m}(t) F_{m}(z)
$$

and use the orthogonality condition in (33) to obtain

$$
(\beta k)^{-1}\left[i b_{n} f\left(k \hat{\tau}_{0}^{y}-l \hat{\tau}_{0}^{x}\right)-d_{n}\right]=\omega_{n}^{-1} \varphi_{n t}-i \varphi_{n},
$$

where

$$
\begin{gathered}
b_{n}=-F_{n}(0), \\
d_{n}(t)=\int_{-h_{0}}^{0} F_{n} \hat{Q} d z .
\end{gathered}
$$

A numerical example illustrates the sensitivity of the forced problem to the imposed scales. We consider a case with exponential stratification [(25a)] with $N_{S}^{2}=7.75 \times 10^{-5}$ and $z_{S}=350 \mathrm{~m}$. In addition, $r=1 \times$ $10^{-4} \mathrm{~m} \mathrm{~s}^{-1}, f=7.3 \times 10^{-5} \mathrm{~s}^{-1}, \beta=2 \times 10^{-11}(\mathrm{~m} \mathrm{~s})^{-1}$, and $h_{0}=4500 \mathrm{~m}$. With scales representative of weather systems, $k=l=3.14 \times 10^{-6} \mathrm{~m}^{-1}$ (Fig. 5, left), modal structures $F_{n}$ are indistinguishable from the inviscid case $G_{n}$, and $b_{0}=-1.49 \times 10^{-2}+i 5.21 \times$ $10^{-5} \mathrm{~m}^{-1 / 2}$ and $b_{1}=-5.41 \times 10^{-2}+i 4.32 \times 10^{-5} \mathrm{~m}^{-1 / 2}$. With scales representative of midocean eddies at $30^{\circ} \mathrm{N}$ (Chelton et al. (2007), results are hardly changed: for $k=l=2.1 \times 10^{-5} \mathrm{~m}^{-1}$ (not shown), modal structures $F_{n}$ are virtually indistinguishable from the inviscid case and $b_{0}=-1.49 \times 10^{-2}+i 3.46 \times 10^{-4} \mathrm{~m}^{-1 / 2}$ and $b_{1}=-5.40 \times 10^{-2}+i 2.82 \times 10^{-4} \mathrm{~m}^{-1 / 2}$. These largely unperturbed modal structures argue strongly that, for the ocean mesoscale, bottom friction cannot account for weakened near-bottom velocities as found by SLF16 (a similar conclusion would be reached even for $r=5 \times 10^{-4} \mathrm{~m} \mathrm{~s}^{-1}$ ). For short scales, approaching the submesoscale, frictional effects become pronounced (Fig. 5, right): when $k=l=1.05 \times 10^{-4} \mathrm{~m}^{-1}$, $b_{0}=-5.87 \times 10^{-4}+i 7.42 \times 10^{-5} \mathrm{~m}^{-1 / 2}$ and $b_{1}=$ $-5.36 \times 10^{-2}-i 2.81 \times 10^{-5} \mathrm{~m}^{-1 / 2}$. For these short scales, the frictional modal modification is large [in accord with (8b)], and the formerly barotropic mode is bottom intensified so that $b_{0}$ becomes two orders of magnitude smaller than for the larger-scale examples. On the other hand, the short-scale baroclinic modes are adjusting toward zero bottom velocity even though the surface amplitude hardly changes; hence, Real $\left(b_{1}\right)$ is largely unchanged. Further, the largest damping of these three examples occurs at intermediate length scales $\left(k=l=2.1 \times 10^{-5} \mathrm{~m}^{-1}\right)$, where $\omega_{I 1}=i 2.34 \times 10^{-9} \mathrm{~s}^{-1}$. When the horizontal length scale is smaller $\left(k=l=1.05 \times 10^{-4} \mathrm{~m}^{-1}\right)$, velocities near the bottom for baroclinic modes are becoming smaller so that damping is smaller $\left(\omega_{I 1}=i 9.84 \times\right.$ $\left.10^{-11} \mathrm{~s}^{-1}\right)$. It thus seems likely that, in the open ocean, modal properties are not strongly affected by bottom friction until very short (less than around $100 \mathrm{~km}$ ) wavelengths are reached.

It is now straightforward to use (36) to compute the ocean's response to a wind stress of the form

$$
\hat{\tau}^{x}=A e^{i \omega t}, \quad \hat{\tau}^{y}=0 .
$$

Sample results, for a 48-day wind forcing period [chosen because Brink (1989) showed that observed temperature fluctuations were driven remotely by the wind at this period where baroclinic Rossby waves are not available], are shown in Fig. 6. The character of the solutions does not change greatly until periods are long enough (order a year or more) to allow near-resonant excitation of baroclinic Rossby modes. For large spatial scales (Fig. 6, left), the response is nearly barotropic and dissipative effects are extremely faint. The velocity shear in the upper $600 \mathrm{~m}$ is consistent, through the thermal wind equation, with the wind driving a temperature response in the upper thermocline. For shorter spatial scales (Fig. 6, right), the pressure response is confined to the upper $400 \mathrm{~m}$ (i.e., a deformation scale) of the water column, and, being surface intensified, the frictional effect is again negligible. 


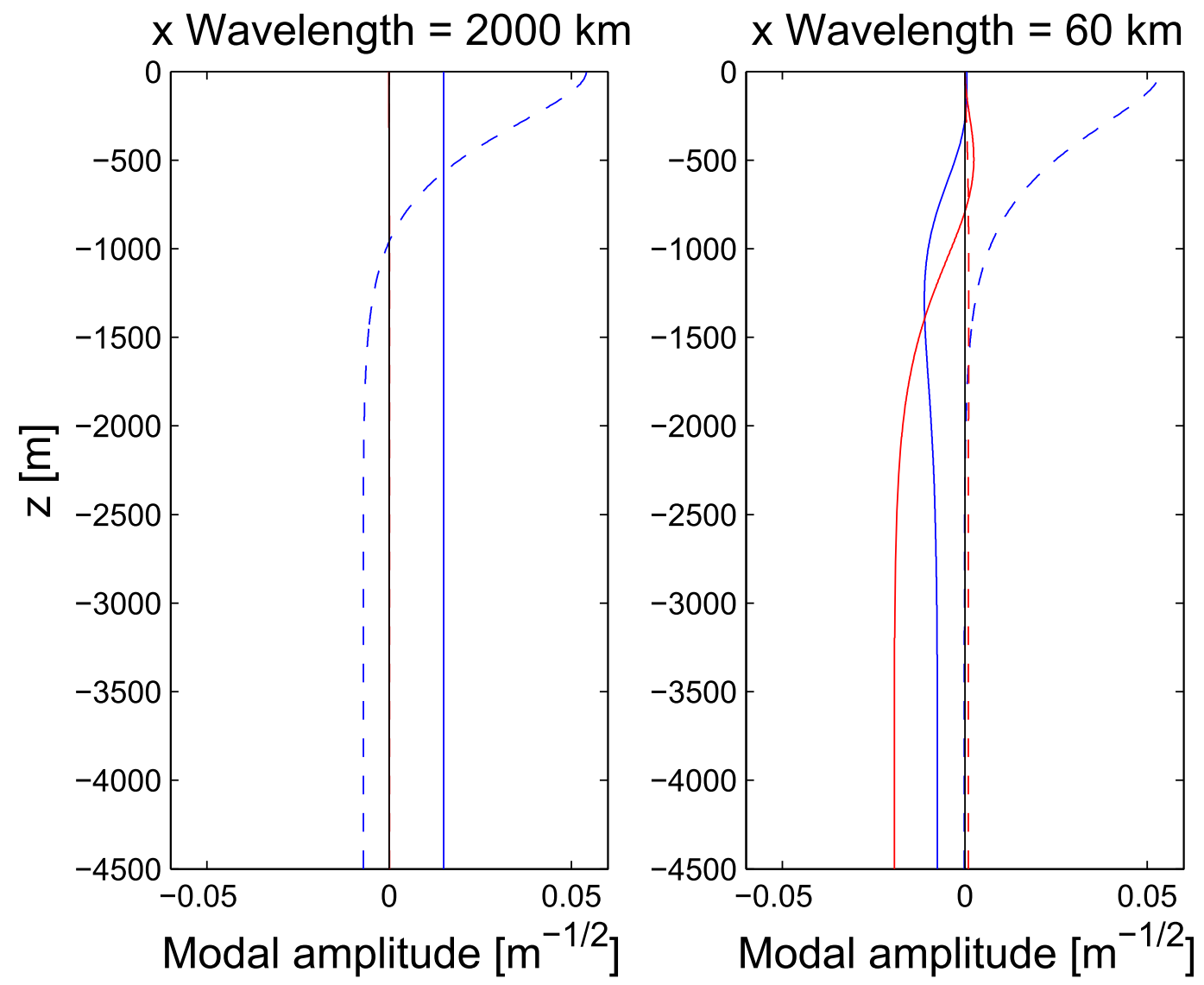

FIG. 5. Modal structures for the two gravest modes with $r=1 \times 10^{-4} \mathrm{~m} \mathrm{~s}^{-1}, f=7.3 \times 10^{-5} \mathrm{~s}^{-1}, \beta=2 \times 10^{-11}(\mathrm{~m} \mathrm{~s})^{-1}$, $h_{0}=4500 \mathrm{~m}$, and exponential stratification $\left(N_{S}^{2}=7.75 \times 10^{-5} \mathrm{~s}^{-2} ; z_{S}=350 \mathrm{~m}\right)$. The solid lines are the gravest mode, and the dashed lines are the next (first baroclinic) mode. Blue is the real part, and red is the imaginary part. The modes are normalized according to (32). (left) Here, $k=l=3.14 \times 10^{-6} \mathrm{~m}^{-1}$. The red lines are indistinguishable from the vertical axis. (right) Here, $k=l=1.05 \times 10^{-4} \mathrm{~m}^{-1}$.

Frictional effects become more obvious at lower frequencies, where differences in $r$ clearly affect response amplitude.

One might ask whether there is really any benefit to be had by expanding the solution to (31) in terms of the frictional vertical modes $F_{n}(z)$ [(4) and (5)]. After all, one could solve the system by expanding in terms of the flat-bottom, inviscid modes $G_{n}(z)$. Comparing solutions using the two approaches, we find that convergence requires the same number of vertical modes using either approach. The advantage of the frictional/ sloping bottom modes lies in (36a), where one simply solves a sequence of uncoupled ordinary differential equations. When the solution is expanded in terms of the inviscid modes, the equivalent of (36a) includes frictional coupling among all the modes. Solving such a system of coupled differential equations for anything except the simplest functional forms of wind stress would be tedious indeed.

\section{Discussion}

One might ask to what extent the above results are relevant to actual oceanographic conditions. Specifically, can frictional effects realistically account for the tendency for many observed subinertial ocean current variations to have an $n=1 / 2$ structure (SLF16)? After all, bottom friction, at some level, is ubiquitous in the ocean, and it would often not entail a sensitivity to wave propagation angle relative to bottom slope. It is, of course, a stretch to apply the present linear results to ocean currents that are known to be nonlinear, but the attempt nonetheless is made. The results in the previous section argue strongly that, for the ocean mesoscale, bottom friction alone cannot account for the SLF16 modal modification, even if a presumably toolarge friction coefficient of $5 \times 10^{-4} \mathrm{~m} \mathrm{~s}^{-1}$ is applied. For substantially shorter wavelengths (i.e., the ocean submesoscale), the modal structures do adjust to have near-zero bottom velocities for the baroclinic modes, 


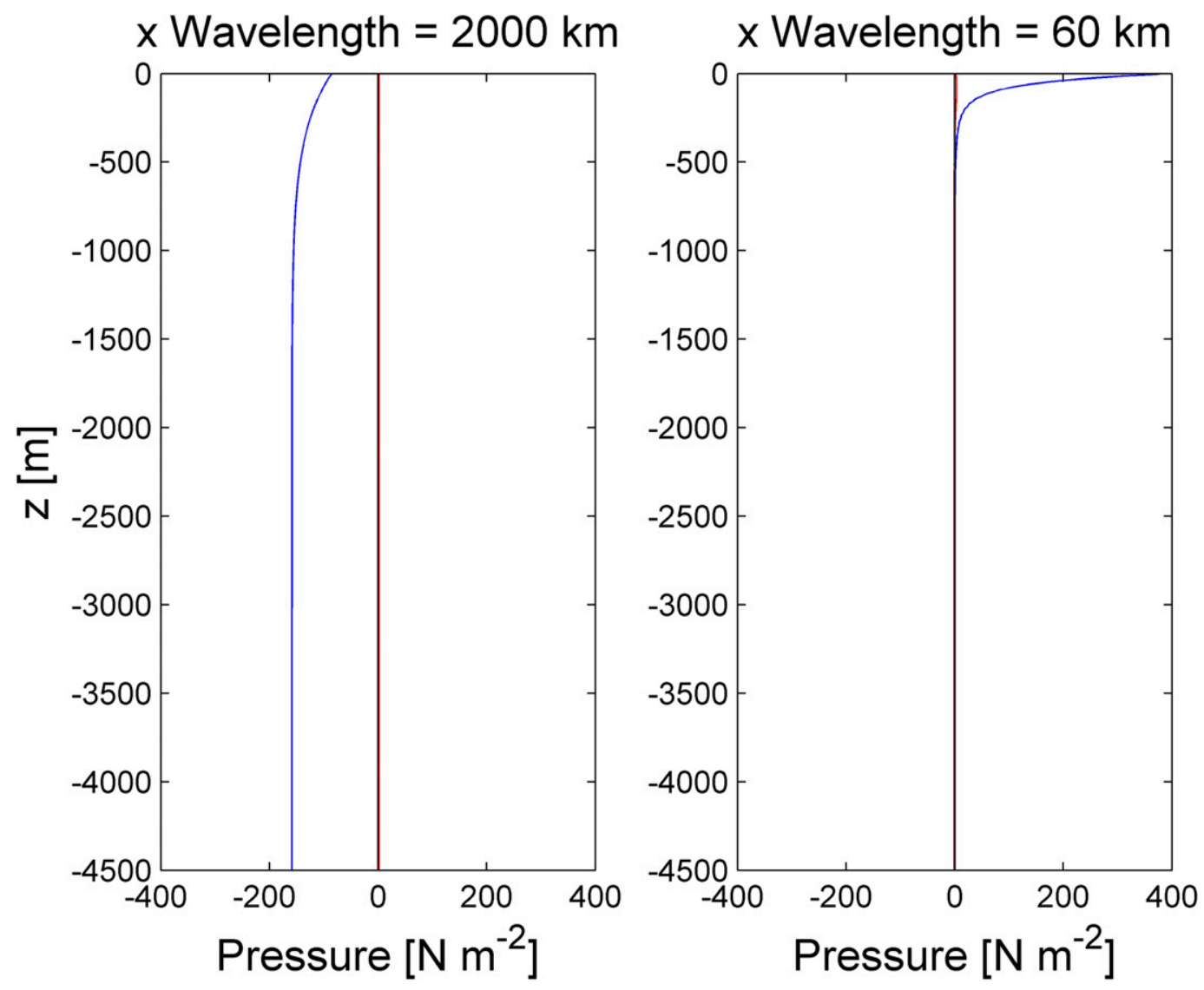

FIG. 6. Pressure response for wind forcing at a 48-day period. Blue curves are the real part of pressure, and red curves are the imaginary part (although the red curves are almost indistinguishable from the vertical axis in both cases). Computed for $r=1 \times 10^{-4} \mathrm{~m} \mathrm{~s}^{-1}, f=7.3 \times 10^{-5} \mathrm{~s}^{-1}, \beta=2 \times 10^{-11}(\mathrm{~m} \mathrm{~s})^{-1}, h_{0}=4500 \mathrm{~m}$, and exponential stratification $\left(N_{S}^{2}=7.75 \times 10^{-5} \mathrm{~s}^{-2} ; z_{S}=350 \mathrm{~m}\right)$. Shown are (left) $k=l=3.14 \times 10^{-6} \mathrm{~m}^{-1}$, and (right) $k=l=1.05 \times$ $10^{-4} \mathrm{~m}^{-1}$.

as expected (Fig. 5). It thus seems that bottom friction by itself is unlikely to distort wave modal structures in the open ocean for the most energetic (mesoscale) eddy length scales. However, this conclusion should be treated as tentative, since it is based entirely on linear physics, while it is likely that nonlinearity plays a substantial role in the actual ocean (e.g., Chelton et al. 2007). Certainly, it has been demonstrated that linearizing about the mean vorticity field associated with a steady flow leads to a modification of modal structures that can either enhance or weaken near-bottom velocities (Killworth et al. 1997). Further, other studies (e.g., Trossman et al. 2017; LaCasce 2017) show that the presence of a sloping or irregular bottom, or of bottom friction, can lead to surface-intensified flow. In contrast to midoceanic conditions, frictional effects in shallower water are relatively more important [as measured by $\left.r /\left(\omega h_{0}\right)\right]$, and stratification is generally stronger in the upper few hundred meters than at abyssal depths. Our analysis suggests that, for water depths shallower than a few hundred meters, bottom friction is clearly expected to affect both linear wave (e.g., Brink 2006) and nonlinear eddy (e.g., Brink 2017) structures.

Perhaps the more unexpected result here is the development of the strongly bottom-trapped, strongly damped mode at short horizontal length scales. A bottom-intensified inviscid mode is of course also found in the presence of a sloping bottom (Rhines 1970), but bottom trapping with a flat bottom was not anticipated. Thus, the near-bottom surface quasigeostrophic (SQG; e.g., Held et al. 1995) phenomenology has been broadened. When the mode is strongly bottom trapped, it is unlikely to be stimulated by a surface wind stress (see section 3), but it could still be readily excited in an initial value problem, in cases with a body forcing, or in problems involving flow over varying bottom topography. Since the bottom-trapped mode is strongly damped, it is most likely to be found close to where it is excited. 
Acknowledgments. We appreciate thoughtful comments from Brian Arbic and Joe LaCasce. Partial funding for this article is provided by the National Science Foundation Physical Oceanography section through Award OCE-1433953.

\section{REFERENCES}

Allen, J. S., 1984: A simple model for stratified shelf flow fields with bottom friction. J. Phys. Oceanogr., 14, 1200-1214, https://doi.org/10.1175/1520-0485(1984)014<1200:ASMFSS> 2.0.CO;2.

Arbic, B. K., and G. R. Flierl, 2004: Baroclinically unstable geostrophic turbulence in the limits of strong and weak bottom Ekman friction: Application to midocean eddies. J. Phys. Oceanogr., 34, 2257-2273, https://doi.org/10.1175/ 1520-0485(2004)034<2257:BUGTIT>2.0.CO;2.

Brink, K. H., 1989: Evidence for wind-driven current fluctuations in the western North Atlantic. J. Geophys. Res., 94, 2029-2044, https://doi.org/10.1029/JC094iC02p02029.

_- 2006: Coastal-trapped waves with finite bottom friction. Dyn. Atmos. Oceans, 41, 172-190, https://doi.org/10.1016/ j.dynatmoce.2006.05.001.

- 2017: Surface cooling, winds, and eddies over the continental shelf. J. Phys. Oceanogr., 47, 879-894, https://doi.org/10.1175/ JPO-D-16-0196.1.

Chapman, D. C., 1987: Application of coastal-trapped wave theory along the California coast. J. Geophys. Res., 92, 1798-1816, https://doi.org/10.1029/JC092iC02p01798.

Chelton, D. B., M. G. Schlax, R. M. Samelson, and R. A. de Szoeke, 2007: Global observations of large oceanic eddies. Geophys. Res. Lett., 34, L15606, https://doi.org/10.1029/2007GL030812.

Held, I. M., R. T. Pierrehumbert, S. T. Garner, and K. L. Swanson, 1995: Surface quasigeostrophic dynamics. J. Fluid Mech., 282, 1-20, https://doi.org/10.1017/S0022112095000012.
Killworth, P. D., D. B. Chelton, and R. A. de Szoeke, 1997: The speed of observed and theoretical long extratropical planetary waves. J. Phys. Oceanogr., 27, 1946-1966, https://doi.org/ 10.1175/1520-0485(1997)027<1946:TSOOAT>2.0.CO;2.

LaCasce, J. H., 2017: The prevalence of oceanic surface modes. Geophys. Res. Lett., 44, 11 097-11 105, https://doi.org/10.1002/ 2017GL075430.

— , and K. H. Brink, 2000: Geostrophic turbulence over a slope. J. Phys. Oceanogr., 30, 1305-1324, https://doi.org/10.1175/ 1520-0485(2000)030<1305:GTOAS > 2.0.CO;2.

Power, S. B., J. H. Middleton, and R. H. J. Grimshaw, 1989: Frictionally modified continental shelf waves and the subinertial response to wind and deep-sea forcing. J. Phys. Oceanogr., 19, 1486-1506, https://doi.org/10.1175/1520-0485(1989)019<1486: FMCSWA $>2.0 . \mathrm{CO} ; 2$.

Rhines, P. B., 1970: Edge-, bottom-, and Rossby waves in a rotating stratified fluid. Geophys. Fluid Dyn., 1, 273-302, https:// doi.org/10.1080/03091927009365776.

Samelson, R. M., 1992: Surface-intensified Rossby waves over rough topography. J. Mar. Res., 50, 367-384, https://doi.org/ $10.1357 / 002224092784797593$.

Sanchez de la Lama, M., J. H. LaCasce, and H. K. Fuhr, 2016: The vertical structure of ocean eddies. Dyn. Stat. Climate Syst., 1, dzw001, https://doi.org/10.1093/climsys/dzw001.

Trossman, D. S., B. K. Arbic, D. N. Straub, J. G. Richman, E. P. Chassignet, A. J. Wallcraft, and X. Xu, 2017: The role of rough topography in mediating impacts of bottom drag in eddying ocean circulation models. J. Phys. Oceanogr., 47, 1941-1959, https://doi.org/10.1175/JPO-D-16-0229.1.

Wright, D. G., and K. R. Thompson, 1983: Time-averaged forms of the nonlinear stress law. J. Phys. Oceanogr., 13, 341-345, https://doi.org/10.1175/1520-0485(1983)013<0341:TAFOTN $>$ 2.0.CO;2.

Wunsch, C., 1997: The vertical partition of oceanic horizontal kinetic energy. J. Phys. Oceanogr., 27, 1770-1810, https:// doi.org/10.1175/1520-0485(1997)027<1770:TVPOOH>2.0.CO;2. 